constipation related symptoms over time $\left(X_{2}=40.87\right.$, $\mathrm{p}<0.001)$. Wilcoxon signed rank tests were used to examine the differences in time points. An MCID difference was found between baseline (median $=14.75, \mathrm{IQR}=12.38-19.75)$ constipation symptoms compared to six weeks (median $=4.75$, $\mathrm{IQR}=2.88-7.38)$ which was significant $(\mathrm{p}<0.001)$. An MCID difference was also found between constipation symptoms at baseline compared to 24 weeks (median $=4.00$, IQR $=1.75-$ $8.44)$ which was significant $(\mathrm{p}<0.001)$. There was no significant difference found between constipation symptoms at six weeks compared to 24 weeks $(p=0.12)$ (figure 1$)$. No adverse events were reported.

Conclusions The study found a significant and clinically meaningful reduction in self- reported constipation symptoms for PwMS after six weeks of using ABFES which was maintained over a 24-week period. High compliance with the treatment over the time period suggests acceptability. The findings are consistent with previous studies and suggest a fully powered controlled trial is required.

\section{P347 PPI REFRACTORY 'REFLUX' SYMPTOMS ARE ASSOCIATED WITH BOWEL SYMPTOM SEVERITY IN PATIENTS WITH CYSTIC FIBROSIS}

Robert Lord, Amanda Brennan, Peter Barry, Jacky Smith, Sharon Archbold, Jane Wych, Peter Whorwell, Andrew Jones, Dipesh Vasant*. Manchester University NHS Foundation Trust, Manchester, UK

\subsection{6/gutjnl-2020-bsgcampus.421}

Introduction Refractory gastrointestinal symptoms are an increasingly recognised area of unmet need, which cause significant morbidity and impair the quality-of-life of patients with Cystic Fibrosis (CF). Recent studies have suggested a high prevalence of both gastro-oesophageal reflux, and bowel symptoms in CF. However the association between reflux symptoms, oesophageal $\mathrm{pH}$ metrics, and overlapping bowel symptoms has not been studied.

Methods CF patients recruited from a tertiary clinic completed validated patient reported outcome measures for reflux (RESQ-7) and functional bowel symptoms (IBS-SSS), before undergoing 24-hour oesophageal $\mathrm{pH}$ and impedance studies. Subjects remained on their regular prescribed acid suppression for the duration of the study. Correlations were examined between reflux symptoms (RESQ-7), bowel symptoms (IBS-SSS) and the oesophageal $\mathrm{pH}$-impendence metrics (total acid exposure, total number of impedance events and Demeester scores), using a Spearman rank. Data are expressed as median and inter-quartile range where appropriate.

Results CF patients $(\mathrm{n}=40$, median age 30 (range 18-48), 28 males, FEV1 50\%, BMI $22 \mathrm{~kg} / \mathrm{m}^{2}$ ), participated in the study. The majority of participants had refractory reflux symptoms $(31 / 40,78 \%)$, and remained on acid suppression during the 24 hour oesophageal $\mathrm{pH} /$ impedance study. Overall, group median oesophageal acid exposure time (oesophageal $\mathrm{pH}<4-2.9 \%$, IQR 7.9), and Demeester scores (11.17, IQR 28.30), were within the normal range, suggesting adequate acid suppression. Strong correlations were seen between total IBS-SSS scores and reflux symptom scores (heartburn severity $r_{s}=0.678, p<0.001$ and frequency $r_{s}=0.649, \quad p<0.001, \quad$ regurgitation severity $\quad\left(r_{s}=0.638\right.$, $\mathrm{p}<0.001)$ and frequency $\left(\mathrm{r}_{\mathrm{s}}=0.627, \mathrm{p}<0.001\right)$. By contrast, no correlation was seen between oesophageal $\mathrm{pH}$-impedance metrics, with either reflux symptom scores $(p=N S)$ or IBSSSS scores $(p=N S)$.

Conclusions This study is the first to demonstrate overlapping foregut and hindgut functional gastrointestinal symptoms in patients with CF using validated patient recorded outcome measures and objective reflux metrics. The correlation between bowel and reflux symptoms, independent of reflux measures, is intriguing, and suggests a possible mechanism of visceral hypersensitivity. The use of gut-brain neuromodulators in this difficult to treat group of patients may therefore have a role and warrants proper investigation.

\section{P348 FURTHER EVIDENCE IN SUPPORT OF BENEFICIAL EFFECTS OF FXR AGONISTS IN BILE ACID DIARRHOEA}

1,2 Julian Walters*. 'Imperial College Healthcare NHS Trust, London, UK; ${ }^{2}$ Imperial College London, London, UK

10.1136/gutjnl-2020-bsgcampus.422

Introduction Primary bile acid diarrhoea (BAD), also known as idiopathic bile acid malabsorption, is a common cause of chronic functional diarrhoea and irritable bowel syndrome with diarrhoea. BAD has been shown to be associated with fatty liver disease including non-alcoholic steatohepatitis (NASH), with shared mechanisms being reported. In our previous proof-of-concept study, patients with BAD had significant clinical improvements in stool type and frequency when treated for two weeks with the first-in-class farnesoid $\mathrm{X}$ receptor (FXR) agonist obeticholic acid (OCA). In a case report using OCA for 6 months, in addition to clinical improvement, normalisation of the diagnostic SeHCAT test was found. The aim of this study was to see if further evidence for benefits of FXR agonists on diarrhoea could be found in the recently published results from the 18 -month interim analysis of a large phase 3 study of OCA in NASH (Younossi ZM, et al. Lancet 2019; 394:2184-2196).

Methods The published results of the interim analysis were analysed independently. Patients with NASH fibrosis $(n=1968)$ had been randomly allocated to placebo, OCA $10 \mathrm{mg}$, or OCA $25 \mathrm{mg}$ daily. Patient-reported adverse reactions had been recorded per protocol. Figures for newly incident events of diarrhoea and constipation were given. The frequencies of these events were compared between the groups.

Results Incidents of diarrhoea were reported in $12 \%$ of patients in the placebo-treated group. These were significantly fewer in both OCA-treatment groups, at $7 \%(\mathrm{p}<0.005$, Fisher's exact tests). Incidents of constipation were the opposite, occurring in 5\% with placebo treatment, 10\% with OCA 10 $\mathrm{mg}$ and $11 \%$ with OCA $25 \mathrm{mg}(\mathrm{p}<0.003)$.

Conclusion Analysis of this trial of OCA in NASH patients indicates that treatment with FXR agonists reduces episodes of diarrhoea and increases constipation. This is presumably by the demonstrated effects of FXR agonists, stimulating FGF19 and reducing bile acid synthesis. Although the primary focus of the trial is on liver fibrosis, these results provide further evidence that FXR agonists can be a therapeutic option in BAD. Trials of FXR agonists in development should include analysis of effects on bowel function, and specifically look at the response in patients with BAD. 